\title{
Ecuador: producción de banano, café y cacao por zonas y su impacto económico 2013-2016
}

\author{
Fecha de recepción :2020-05-17 • Fecha de aceptación: 2020-07-15 • Fecha de publicación: 2020-09-10
}

Lady Andrea León Serrano ${ }^{1}$ Universidad Técnica de Machala, Ecuador

Ilady@utmachala.edu.ec

https://orcid.org/0000-0001-5472-140X

Amparo Marisol Matailo Pinta ${ }^{2}$ Universidad Técnica de Machala, Ecuador amatailo@utmachala.edu.ec https://orcid.org/0000-0001-5223-9732

Aida Andreina Romero Ramón ${ }^{3}$ Universidad Técnica de Machala, Ecuador aromero@utmachala.edu.ec https://orcid.org/0000-0001-7493-8681

Cecilia Alexandra Portalanza Chavarría ${ }^{4}$ Universidad Espíritu Santo, Ecuador aportalanza@uees.edu.ec http://orcid.org/0000-0002-9782-5089

\section{Resumen}

El objetivo de la investigación consiste en identificar la zonificación de mayor producción de Banano, Café y Cacao y su impacto económico en el Ecuador, período 2013-2016. La planificación zonal es un modelo de gestión con propuestas de desarrollo para los diferentes sectores, principalmente el productivo por el efecto vinculante, permitiendo la descentralización de funciones y la especialización de áreas de influencia para el respectivo fortalecimiento territorial. Los datos fueron obtenidos 
del Banco Central del Ecuador planteando el estudio con un enfoque cuantitativo y descriptivo, los valores fueron procesados estadísticamente, obteniendo como principales resultados que la zona 5 comprendido por Santa Elena, Guayas, Bolívar, Los Ríos y Galápagos son las de mayor producción seguido de la zona 7 con menor porcentaje, representando ser el segundo rubro de la economía nacional, por lo tanto, las principales conclusiones determinan a una economía dependiente del sector agropecuario por el aporte económico brindado en los niveles de producción y exportaciones en el Producto Interno Bruto mediante la consolidación de políticas económicas y públicas para las zonas ecuatorianas.

\title{
Palabras clave: producción, planificación zonal, banano, café, cacao, Ecuador.
}

\begin{abstract}
The objective of the research is to identify the zoning of the highest production of Banana, Coffee and Cacao and its economic impact in Ecuador, period 2013-2016. The zonal planning is a management model with development proposals for the different sectors, mainly productive due to the binding effect, allowing the decentralization of functions and the specialization of areas of influence for the respective territorial strengthening. The data were obtained from the Central Bank of Ecuador proposing the study with a quantitative and descriptive approach, the values were statistically processed, obtaining the main results when determining that zone 5 comprised of Santa Elena, Guayas, Bolívar, Los Ríos and Galápagos are the of greater production followed by zone 7 with a lower percentage, representing being the second largest item in the national economy, therefore, the main conclusions determine an economy dependent on the agricultural sector due to the economic contribution provided in the levels of production and exports in the Gross Domestic Product through the consolidation of economic and public policies for the Ecuadorian zones.
\end{abstract}

Keywords: production, zonal planning, banana, coffee, cocoa, Ecuador. 


\section{Introducción}

El aumento de la productividad del sector agropecuario ha permitido ampliar la fuerza laboral en los diferentes sectores económicos, han transcurrido varias décadas para establecer la relación entre agricultura y crecimiento económico global, los cambios acontecidos desde el inicio de la era industrial establecieron cambios estructurales en el desarrollo agrícola. Las estrategias de desarrollo económico han favorecido de manera marcada al sector industrial, América Latina y algunos países de Asia manifestaron que el papel de la agricultura se sustenta en proveer productos para el consumo urbano y exportación. Varios observadores han recalcado que el buen uso de los recursos primarios lleva la creación de riqueza nacional con ayuda de la intervención gubernamental (Food and Agriculture Organization of the United Nations (FAO), 2017).

Los mecanismos de crecimiento económico para una expansión global llevan a fortalecer el sector primario por la dependencia de la agricultura como primer ingreso local y nacional. Los países europeos, por su clima frío, han sido los menos favorecidos en la especialización de cierto tipo de frutas, generando una fortaleza para los países tropicales identificados, en su mayoría, en el cono Sur. Ecuador es un país agroexportador desde antes de ser considerada República, la experiencia del boom bananero y cacaotero condujeron a un aprovechamiento de las ventajas comparativas para ser una de las economías con más recursos en la década de los cincuenta hasta los setenta, ubicándolo en los principales competidores de fruta de banano, siguiendo el cacao y más adelante el café.

Tradicionalmente la agricultura ha sustentado la estructura productiva con especialidad en las exportaciones en la Costa y para el consumo interno en la Sierra; el énfasis en la producción agropecuaria exportable permitió cambios en la diversificación de los productos tradicionales, las perspectivas se mantuvieron en un desarrollo rural económicamente de bienestar social y económico, a pesar de una demanda de alimentos creciente, el tipo de productos fueron acoplados al estilo de vida de la población urbana y rural (Ministerio de Agricultura, Ganadería, Acuacultura y Pesca (MAGAP), 2017).

Ecuador es considerado uno de los mayores productores de banano, café y cacao, esto significó una oportunidad para reorganizar la agenda territorial con alcance a rentalizar la productividad y focalización de apoyo en los sectores de cosecha y exportación. De acuerdo a la Secretaria Nacional de Planificación y Desarrollo del Ecuador (SENPLADES, 2015), el proceso de zonificación es parte de las reformas políticas en articular la acción pública estatal como una forma efectiva y eficiente de acortar las distancias entre gobernados-gobernantes y generando inversión en el sector agro. Este modelo de Estado, con estructuras regionales, comprende la planificación territorial para propiciar una base administrativa que contribuya a la mejora de la calidad de vida socioeconómica de la población en que la fuerza laboral trabaja en su mayoría en actividades agrícolas y comerciales. Las fuentes de plantaciones de banano, café y cacao de pequeños y medianos productores son parte del Plan Regional de Desarrollo y Ordenamiento Territorial como un incentivo para cada una de las zonas donde se produce y exporta.

La importancia del tema radica en el estudio de los aspectos que propiciaron el aumento de la 
producción de las frutas objeto del análisis, como aporte al Producto Interno Bruto y reactivación económica interna, considerando las zonas que fomentaron mayores ingresos económicos siendo zonas especiales de desarrollo económico, por tal motivo, el objetivo de la investigación consiste en identificar la zonificación de mayor producción de banano, café y cacao, además de su impacto económico en el Ecuador durante el período 2013-2016. La metodología aplicada es cuantitativa mediante la obtención de Tablas Anova, un modelo estadístico acertado para este tipo de estudio planteando 7 zonas territoriales, para luego obtener un determinante coeficiente de productividad complementado con investigación descriptiva que contrasta la teoría con los resultados obtenidos.

Los resultados de la investigación se explican por las circunstancias favorables de las zonas: tipo suelo, clima y recursos destinados, otro aspecto fundamental se debe a la potencialidad del sector primario en el Ecuador, por lo tanto, los datos obtenidos explican que la zona 5 y 7 son de mayor productividad y exportaciones, ocasionando un significativo impacto en el progreso económico. La consolidación de políticas económicas y públicas han sido base para el mejoramiento socioeconómico de dichas zonas del país.

\section{Producción agropecuaria y la incidencia económica}

El papel del sector agropecuario en la economía nacional ha quedado evidenciado a lo largo de la historia económica y social del Ecuador. Para Gonzabay (2013), actualmente cubre el 95\% de la demanda interna de los alimentos que consume la población; genera empleo al $25 \%$ de la población económicamente activa (PEA), después del petróleo es el más importante generador de divisas; sin embargo, la balanza comercial del sector es altamente favorable y su aporte en el PIB es relevante, ante esto, Pino, Aguilar, Apolo y Sisalema (2018) explica que la tasa de crecimiento económico extendió algunos picos importantes en los años 2013 y 2014 debido a la estabilidad del nuevo sistema monetario, incidiendo en el incremento de la inversión real, posteriormente este indicador cae en el 2015 y 2016 por el complejo entorno nacional e internacional que se desenvolvió el país, afectado por la caída del precio del crudo, apreciación del dólar ocasionando una ligera recesión e incremento del desempleo.

Las experiencias en el sector primario comenzaron a modificar las iniciativas de las políticas públicas en los sembríos, empleo, sector industrial y el beneficio de la inversión en el agro comercio. Dichas políticas públicas se enmarcaron en la regulación de los mercados en temas de comercialización, intervención del Estado en la regulación, control en los intercambios y transacciones económicas, fijación de políticas de precios, protección integral del sector agrario al garantizar la alimentación de todos los ecuatorianos mediante la soberanía alimentaria, sustitución de importaciones y cambios en la matriz productiva con énfasis en la planificación por zonas territoriales (Andrade, D y Andrade, M 2018). La toma de decisiones en cada una de las zonas comprendidas por las provincias de la Sierra, Costa, Oriente presentan aplicaciones generales valorando la especialidad de los bienes primarios. Según Pino, y otros (2018) el PIB de la agricultura, que cuantifica el tamaño del sector agropecuario, sostuvo un incremento durante el tiempo de estudio, se atribuye a los proyectos del Ministerio de Agricultura, Ganadería y Pesca de alto rendimiento en los programas de reactivación bananera, cafetera y cacaotera en la implementación de precios referenciales para los cultivos, por lo cual el PIB agropecuario fue 
tomando un rol de vital importancia económica y social.

Los volúmenes de producción experimentados dan a conocer el nivel de representatividad del sector en la economía nacional, ante esto, se ha visto limitada por el desarrollo de otros sectores (Tabla 1)

Tabla 1.

PIB agropecuario

\begin{tabular}{lll}
\hline Año & $\begin{array}{l}\text { PIB Agropecuario } \\
\text { (Millones de USD) }\end{array}$ & $\begin{array}{l}\text { PIB Agropecuario/PIB Total } \\
(\%)\end{array}$ \\
\hline 2013 & 5,770 & 8.5 \\
\hline 2014 & 6,026 & 8.6 \\
\hline 2015 & 6,257 & 9.1 \\
\hline 2016 & 6,430 & 9.3 \\
\hline
\end{tabular}

Fuente: Banco Central del Ecuador (2017)

\section{Producción de banano, café y cacao en el Ecuador}

En algunos cantones de Manabí, como Jipijapa, se caracterizan por la abundante producción del grano de café, siendo el período de estudio de gran representatividad en exportaciones. Ecuador cuenta con buena parte de terrenos que se dedican a producir café, esto genera la utilización de mano de obra tanto directa como indirecta.

De esta manera, Briones (2018) menciona que:

"Las provincias que reúnen estas condiciones y se consideran productoras de este tipo de café son: Manabí, Loja, El Oro, Carchi, Zamora Chinchipe, Morona Santiago, Pastaza, Bolívar, Chimborazo, Azuay, Cañar, Cotopaxi, Pichincha, Santo Domingo, Imbabura, Los Ríos, Guayas, Esmeraldas y Galápagos” (pág. 34).

Sin embargo, Zaruma, cantón Orense, tiene el mejor café categorizado a nivel nacional. El cacao es uno de los productos emblemáticos, la distribución del cultivo se ha expandido y ocupado nuevas zonas geográficas concentrándose mayoritariamente en la región costa del Ecuador, tales como Los Ríos, Manabí, Esmeraldas y El Oro y en la sierra Cotopaxi, Bolívar y Cañar, existen otras provincias con potencial poco explotado como Napo, Orellana y Sucumbíos. En los años 2012 hasta 2016, el aporte comercial exterior de cacao en grano al PIB fue de 0,77\% en promedio, una participación que fomentó el interés de seguir reformando los planes de desarrollo al sector cacaotero (Loayza, 2018).

El banano forma parte de los tres productos con mayor volumen de exportación, los tributos generados han registrado saldos positivos en la balanza comercial, más del $30 \%$ de la oferta mundial del banano proviene de Ecuador, país destacado como el principal exportador en el mundo desde el comienzo de los intercambios comerciales. A pesar de haber experimentado situaciones de caída en la producción por fenómenos naturales, el banano sigue teniendo 
características singulares y altos estándares de calidad, manteniéndose en varios países como una de las frutas de gran consumo y dieta diaria (El Productor, 2017). En la Tabla 2 se detallan las principales provincias con alta producción de banano en el 2016, resaltando Los Ríos con 43.23\% y Guayas obteniendo el $32.76 \%$.

Tabla 2.

Producción de banano por principales provincias

\begin{tabular}{llll}
\hline Año & Provincia & Superficie cosechada (ha) & Porcentaje Nacional \\
\hline 2016 & Los Ríos & 61,937 & $43.23 \%$ \\
\cline { 2 - 4 } & Guayas & 48,805 & $32.76 \%$ \\
\cline { 2 - 4 } & El Oro & 42,340 & $16.47 \%$ \\
\cline { 2 - 4 } & Otras & 27,254 & $7.54 \%$ \\
\cline { 2 - 4 } & Total General & $\mathbf{1 8 0 , 3 3 6}$ & $\mathbf{1 0 0} \%$ \\
\hline
\end{tabular}

Fuente: INEC (2016)

Es importante recalcar las exportaciones totales de Banano, por país se destinaron durante el 2013 hasta el 2017 a la Unión Europea, siendo el socio importador con más de 3,685,390 miles de millones USD, seguido de Rusia con 2,545,742 miles de millones USD y Estados Unidos en 3,139,680 miles de millones USD.

El sector agropecuario ha demostrado el incipiente papel en la economía ecuatoriana por la dependencia de los productos objeto de estudio, la creciente producción ha permitido ser el abastecedor de materia prima para otros destinos del mundo y ser dependientes. Durante los últimos veinte años el crecimiento de este rubro ha sostenido a la economía ante eventuales crisis económicas; sin embargo, es necesario conocer el comportamiento futuro del comercio exterior agropecuario para que no se modifique significativamente su estructura.

\subsection{Planificación zonal ecuatoriana}

La propuesta de zonificación como alternativa de desarrollo es parte de la reforma política 20072009 , esta promueve un modelo de gestión endógeno con inserción estratégica y soberana en el sistema nacional, basada en la producción sistémica que garantiza la soberanía alimentaria y el fortalecimiento de actividades generadoras de valor. La construcción de este modelo de Estado comprende tres desafíos: la territorialización de la política pública; planificación en el uso territorial, y el fomento de una estructura administrativa de gestión pública. Dichas regiones administrativas se distribuyeron en Región 1: Esmeraldas, Carchi, Imbabura y Sucumbíos; Región 2: Pichincha, Napo y Orellana; Región 3: Pastaza, Cotopaxi, Tungurahua y Chimborazo; Región 4: Manabí, Santo Domingo de los Tsáchilas; Región 5: Península de Santa Elena, Guayas, Los Ríos y Bolívar; Región 6: Cañar, Azuay y Morona Santiago; Región 7: El Oro, Loja y Zamora; Archipiélago de Galápagos (Secretaría Nacional de Planificación y Desarrollo del Ecuador (SENPLADES), 2012).

La planificación estratégica de SENPLADES (2018) permite la modificación mediante Decreto Ejecutivo No 35728, publicado en el Registro Oficial No. 205 del 2 de junio de 2010, por 
denominación de zonas administrativas de planificación, estableciéndose en nueve, es decir, se aumenta la Zona 8: Cantones Guayaquil, Durán y Samborondón y Zona 9: Distrito Metropolitano de Quito.

Las principales actividades de las zonas se han fundamentado en el desarrollo agropecuario, producción de cultivos distribuidos con diversificación de espacios geográficos que permitan sembrar cantidades suficientes con destino al consumo nacional y adicionalmente para la exportación, permitiendo la ampliación del abanico de cultivos en las diferentes regiones del país, prioritariamente las rurales (SENPLADES, 2012).

Las iniciativas mediante la adecuada gestión del territorio han consolidado espacios de gobernabilidad para efectivizar la productividad agrícola de las zonas del Ecuador y el crecimiento económico que contrae la inversión en el sector primario, por tal motivo, realizar un estudio de la producción distribuida por regiones permite comprender los niveles de participación de cada provincia y seguir potencializando el sector bananero, cafetalero y cacaotero por parte del Estado.

\section{Metodología}

Una vez explicado de manera teórica el tema de estudio, podemos indicar el método planteado en la investigación, inicialmente, según Plata (2019), la metodología es el sistema general de conceptos, principios, métodos, técnicas de estudio, procedimientos para organizar, formular y resolver problemas científicos, sociales y económicos.

El método utilizado es no experimental, ya que este no permite la manipulación de variables, sino únicamente la observación y análisis de los resultados obtenidos de cálculos expuestos (Coria, Torres y Roman, 2014). También es descriptiva por la recopilación de datos necesarios para alcanzar el objetivo que sirven para comprender la productividad del banano, café y cacao 20132016 por zonas o territorios y la incidencia económica.

El desarrollo metodológico plantea un proceso estadístico para un tipo de relación entre variables endógenas y exógenas para la descripción de características de la muestra (Villavicencio, 2013). Así, el método es cuantitativo, de selección subjetiva e intersubjetiva de indicadores de ciertos elementos de procesos, hechos, estructuras y personas. Estos elementos no conforman en su totalidad, los procesos o las personas, de aquí se deriva el debate entre los cuantitativistas que nunca ven un fenómeno integrado, sino siempre conjuntos de partículas de los fenómenos relacionados con la observación (Cadena, y otros, 2017), que procede en la aplicación de procedimientos estadísticos con categorización de variables (Trívez, 2015).

Los datos obtenidos corresponden a la información del Banco Central del Ecuador, clasificado por provincias y zonas.

La categorización de variables es el proceso por el cual especificamos cuáles serán las categorías de interés, a su vez, (...) son las diferentes posibilidades de variación que una variable puede tener (Cazu, 2004), mediante la siguiente Tabla 3 se ubica el nombre de la variable, tipo, indicador al cual pertenece y la unidad de medida. 
Tabla 3.

Categorización de variables

\begin{tabular}{llll}
\hline Variable & Característica & Indicador & Unidad de medida \\
Producción agrícola & Cuantitativa & Producción de banano & USD \\
& & Producción de café & USD \\
& & Producción de cacao & USD \\
Provincias por zonas & Cualitativa & Enumeradas por zonas & Índice 1-7 \\
\hline
\end{tabular}

Fuente: Banco Central del Ecuador (2017)

Mientras que en la Tabla 4 se evidencia el desagregado del indicador (enumerado por zonas con Unidad de medida=Índice 1-7), comprendiendo que la planificación zonal tiene un aporte significativo en el progreso económico. Para el presente estudio se consideró la Planificación Zonal 2007-2009 con siete zonas administrativas.

Tabla 4.

Distribución provincial por zonas

\begin{tabular}{lllllll}
\hline Zonas de Ecuador & & & & \\
\hline Zona 1 & Zona 2 & Zona 3 & Zona 4 & Zona 5 & Zona 6 & Zona 7 \\
\hline Esmeraldas & Pichincha & Cotopaxi & Manabí & Santa Elena & Cañar & El Oro \\
\hline Imbabura & Napo & Tungurahua & $\begin{array}{l}\text { Santo } \\
\text { Domingo }\end{array}$ & Guayas & Azuay & Loja \\
\hline Carchi & Orellana & Chimborazo & & Bolívar & Morona & Zamora \\
& & Pastaza & & Los Ríos & & Chinchipe \\
\hline Sucumbíos & & & & Galápagos & & \\
\hline
\end{tabular}

Fuente: Banco Central del Ecuador (2017)

En base a lo anterior, se establece la siguiente ecuación tomando en cuenta las zonas de Ecuador:

Ecuación (1)

$y=\beta 1+\beta 2+\beta 3+\beta 4+\beta 5+\beta 6+\beta 7$

Representando:

$\mathrm{y}=$ producción de banano, café y cacao

$\beta 1=$ Producción de la zona 1

$\beta 2=$ Producción de la zona 2

$\beta 3=$ Producción de la zona 3

$\beta 4=$ Producción de la zona 4 
$\beta 5=$ Producción de la zona 5

$\beta 6=$ Producción de la zona 6

$\beta 7=$ Producción de la zona 7

El software estadístico permitió realizar tablas ANOVA tomando en cuenta que, si existen 7 zonas, el resultado de los coeficientes es la productividad. Este proceso se realizó con los años de estudio, para luego reunir los coeficientes y ordenarlos para mejor entendimiento de los resultados.

\section{Resultados}

En este primer apartado identificaremos la zona con mayor productividad de banano, café y cacao, utilizando los datos de 24 provincias agrupadas por 7 zonas, para eso fue necesario realizar regresiones individuales por cada uno año de estudio 2013, 2014, 2015 y 2016.

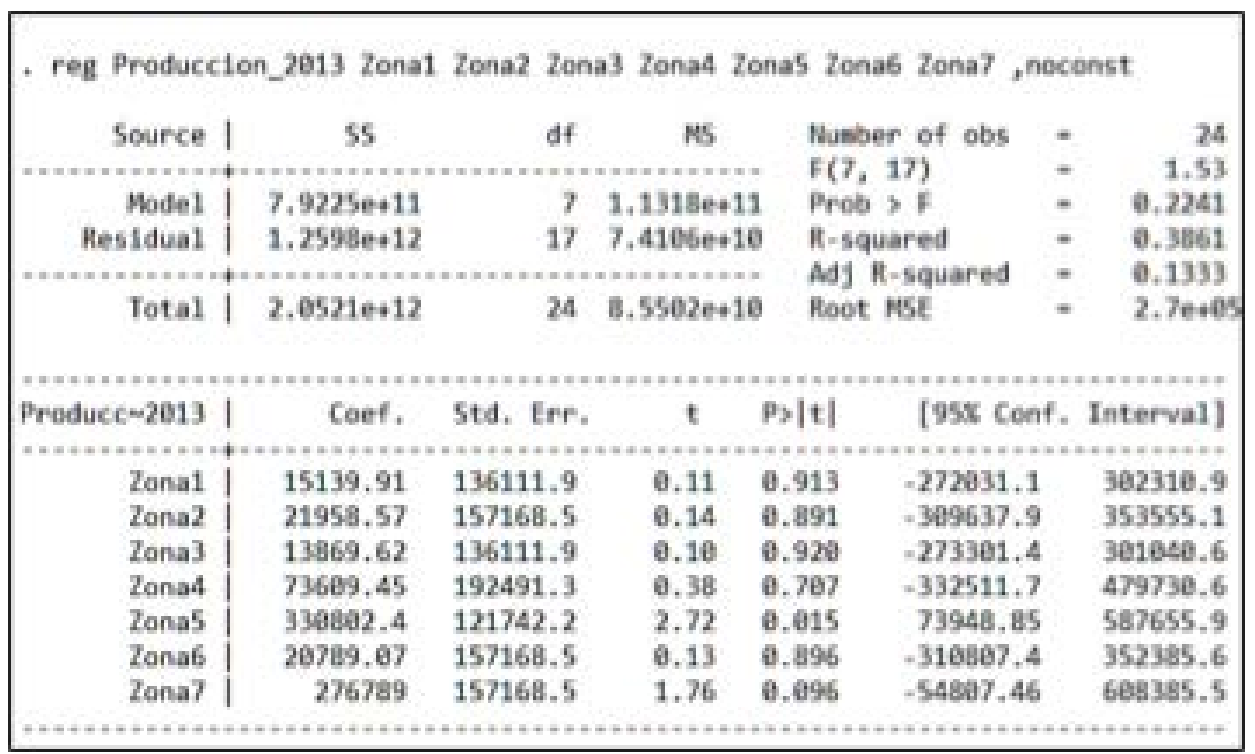

Figura 1. Tabla ANOVA-Producción año 2013

Fuente: elaboración propia con datos del Banco Central del Ecuador (2017)

Reemplazando la ecuación 1 con los datos de la Figura 1, obtenemos la siguiente fórmula:

Ecuación (2)

$y=\beta 1+\beta 2+\beta 3+\beta 4+\beta 5+\beta 6+\beta 7$

Producción $2013=15139,91$ Zona1+21958,57 Zona2+13869,62 Zona3+73609,45 Zona4+330802,40

Zona5+20789,07 Zona6+276789,00 Zona7 


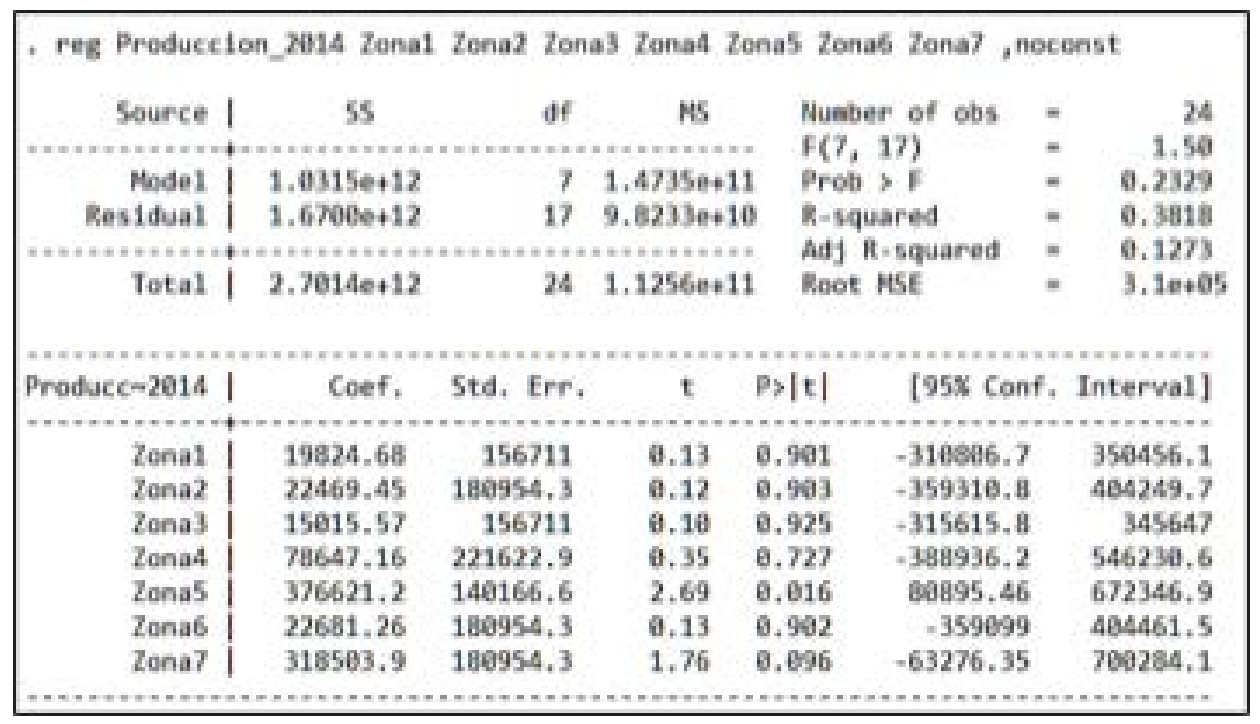

Figura 2. Tabla ANOVA-Producción año 2014

Fuente: elaboración propia con datos del Banco Central del Ecuador (2017)

Reemplazando la ecuación 1 con los datos de la Figura 2, obtenemos la siguiente fórmula:

Ecuación (3)

$y=\beta 1+\beta 2+\beta 3+\beta 4+\beta 5+\beta 6+\beta 7$

Producción $2014=19824,68$ Zona1+22469,45 Zona2+15015,57 Zona3+78647,16 Zona4+376621,20 Zona5+22681,26 Zona6+318503,90 Zona7

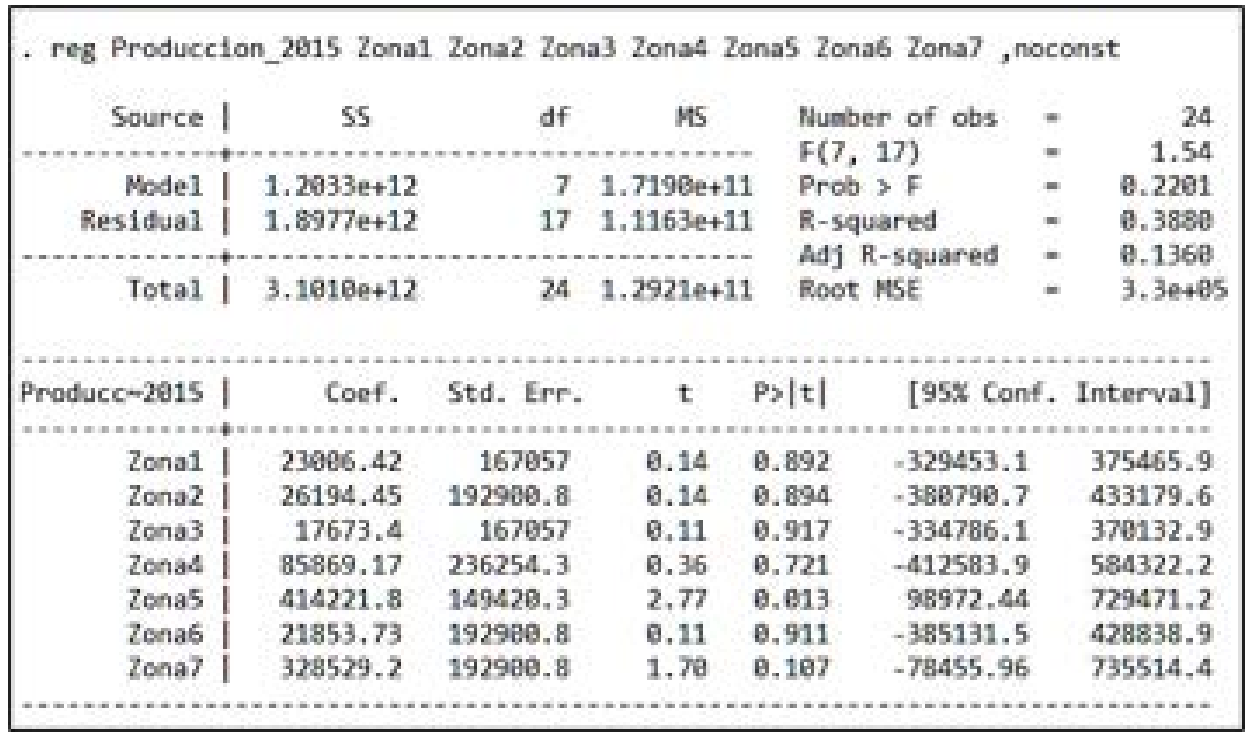

Figura 3. Tabla ANOVA-Producción año 2015

Fuente: elaboración propia con datos del Banco Central del Ecuador (2017) 
Reemplazando la ecuación 1 con los datos de la Figura 3, obtenemos la siguiente fórmula:

Ecuación (4)

$y=\beta 1+\beta 2+\beta 3+\beta 4+\beta 5+\beta 6+\beta 7$

Producción $2015=23006,42$ Zona1+26194,45 Zona2+17673,40 Zona3+85869,17 Zona4+414221,80

Zona5+21853,73 Zona16+328529,20 Zona7

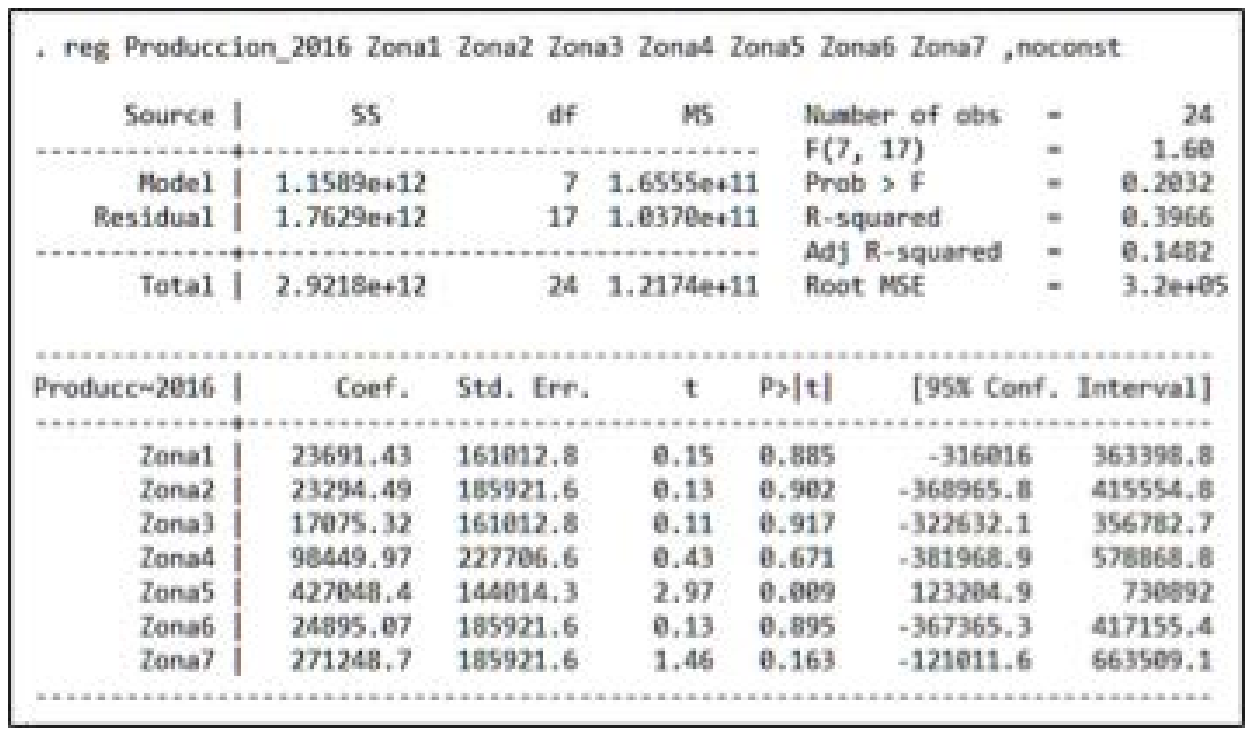

Figura 4. Tabla ANOVA-Producción año 2016

Fuente: elaboración propia con datos del Banco Central del Ecuador (2017)

Reemplazando la ecuación 1 con los datos de la Figura 4, obtenemos la siguiente fórmula:

Ecuación (5)

$$
\mathrm{y}=\beta 1+\beta 2+\beta 3+\beta 4+\beta 5+\beta 6+\beta 7
$$

Producción 2016 = 23691,43 Zona1+23294,49 Zona2+17075,32 Zona3+98449,97 Zona4+427048,40 Zona5+24895,07 Zona6+271248,70 Zona7

Para la interpretación de las Figuras 1,2,3 y 4 se han considerado los coeficientes que determinan la producción de banano, café y cacao para cada zona, observando la de mayor aporte productivo es la Zona 5 durante los años 2013, 2014, 2015 y 2016 comprendida por Santa Elena, Guayas, Bolívar, Los Ríos y Galápagos (2013=330802,4; 2014=376621.2; 2015=414221.8 y 2016=427048.4); luego sigue la Zona 7. El crecimiento productivo económico experimentado por los dos sectores se debe a la dinamización del sector agrícola con un avance importante en el aumento del empleo en concordancia con la efectividad empresarial.

En las siguientes tablas se analiza los coeficientes de cada zona por año, esto permite comprender el impacto de la producción de banano, café y cacao en la economía del país. 
Con los datos de la Tabla 5 se verifica la curva de la Figura 5 con una línea de tiempo, observando que la productividad total de las frutas de estudio en las zonas es constante por un efecto creciente moderado desde el 2013 al 2015; luego, hasta el 2016, existe una leve disminución de productividad por los altos costos de mano de obra, insumos y el costo de transportación de productos internos.

Tabla 5.

Coeficiente ANOVA: Producción por años

\begin{tabular}{lllll}
\hline $\begin{array}{l}\text { Provincias } \\
\text { por Zonas }\end{array}$ & $\begin{array}{l}\text { Produccion-2013 } \\
\text { (Miles de Millones } \\
\text { USD) }\end{array}$ & $\begin{array}{l}\text { Produccion-2014 } \\
\text { (Miles de Millones } \\
\text { USD) }\end{array}$ & $\begin{array}{l}\text { Produccion-2015 } \\
\text { (Miles de } \\
\text { Millones USD) }\end{array}$ & $\begin{array}{l}\text { Produccion-2016 } \\
\text { (Miles de } \\
\text { Millones USD) }\end{array}$ \\
\hline Zona 1 & 15139,91 & 19824,68 & 23006,42 & 23691,43 \\
\hline Zona 2 & 21958,57 & 22469,45 & 26194,45 & 23294,49 \\
\hline Zona 3 & 13869,62 & 15015,57 & 17673,40 & 17075,32 \\
\hline Zona 4 & 73609,45 & 78647,16 & 85869,17 & 98449,97 \\
\hline Zona 5 & 330802,40 & 376621,20 & 414221,80 & 427048,40 \\
\hline Zona 6 & 20789,07 & 22681,26 & 21853,73 & 24895,07 \\
\hline Zona 7 & 276789,00 & 318503,90 & 328529,20 & 271248,70 \\
\hline Total & 752958,02 & 853763,22 & 917348,17 & 885703,38 \\
\hline
\end{tabular}

Fuente: Banco Central del Ecuador (2017)

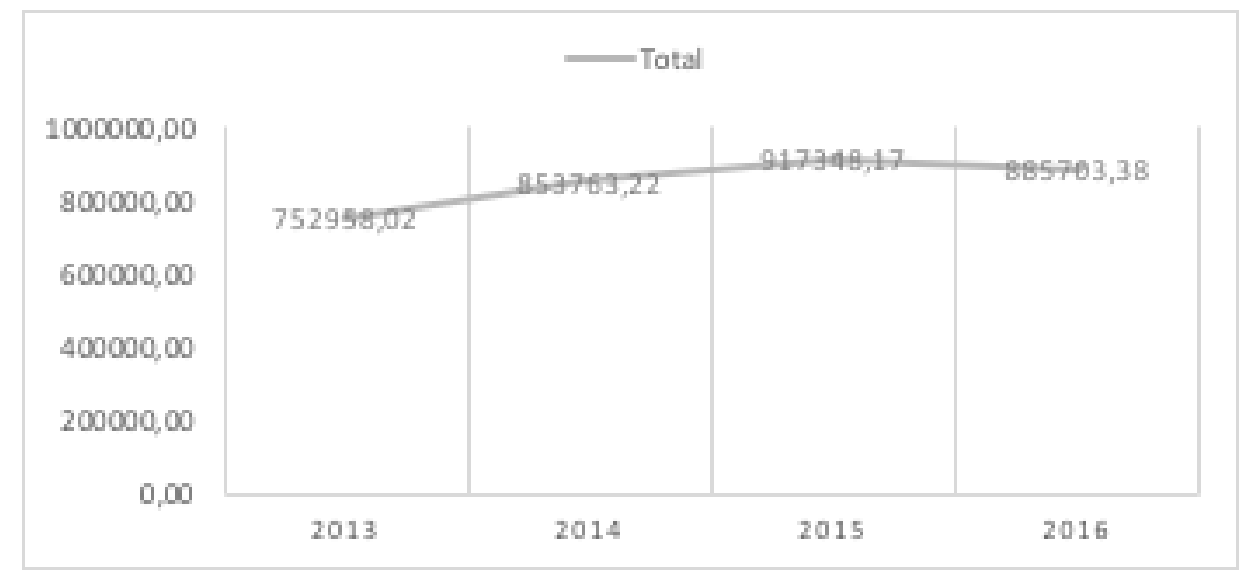

Figura 5. Variación de la producción total de Banano, Café y Cacao, 2013-2016

Fuente: Banco Central del Ecuador (2017)

En la siguiente Tabla 6 se evidencian los valores de producción por zonas desde el 2013 hasta el 2016, siendo estas las fechas planteadas de la investigación. 
Tabla 6.

Producción general por Zonas

\begin{tabular}{lllll}
\hline $\begin{array}{l}\text { Provincias por } \\
\text { zonas }\end{array}$ & Produccion-2013 & Produccion-2014 & Produccion-2015 & Produccion-2016 \\
\hline Zona 4 & 73609,45 & 78647,16 & 85869,17 & 98449,97 \\
\hline Zona 5 & 330802,40 & 376621,20 & 414221,80 & 427048,40 \\
\hline Zona 7 & 276789,00 & 318503,90 & 328529,20 & 271248,70 \\
\hline Zonas 1-2-3-6 & 71757,17 & 79990,96 & 88728,00 & 88956,31 \\
\hline
\end{tabular}

Fuente: Banco Central del Ecuador (2017)

Mientras que en la Figura 6 se muestra el porcentaje de participación producción por zonas, para esta fue necesario agrupar en un solo valor la zona 1, 2 y 3; mientras se mantienen los valores individuales en la zona 4, 5 y 7, observando que el mayor peso de rendimiento recae sobre el sector 5 con un $44 \%$ y $37 \%$ la zona 7 ; la influencia destaca una vez más que las localidades que forman cada zona aportan con un incremento fundamental en el Producto Interno Bruto del país, la dinámica se estableció en un aumento de la producción mejorando la inversión e incremento de la movilidad del comercio agrícola, siendo una las fuentes de mayor interés exterior para los acuerdos comerciales.

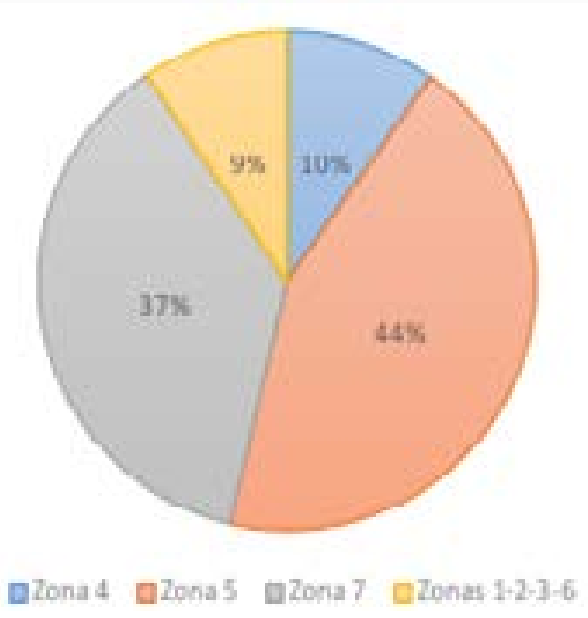

Figura 6. Participación en porcentaje de producción por zonas

Fuente: Banco Central del Ecuador (2017)

Analizando las Figuras expuestas (valores de la Tabla 5), se realizaron para comprender el comparativo de los niveles de productividad otras gráficas, en la Figura 7 se visualiza claramente las zonas 5, 7 y 4 con mayor especialidad en rendimiento agropecuario de las frutas de estudio en el período descrito marcando un auge de medidas por las aperturas comerciales fuera del país que aportaron generosamente al desarrollo económico internacional; mientras las zonas 1, 2, 3 y 6 han tenido bajos niveles de productividad en ciertas Provincias debido a su baja producción, pues se especializan en otros productos la mayoría, sin embargo los recursos generados han servido de apoyo a los diferentes sectores agropecuarios. 


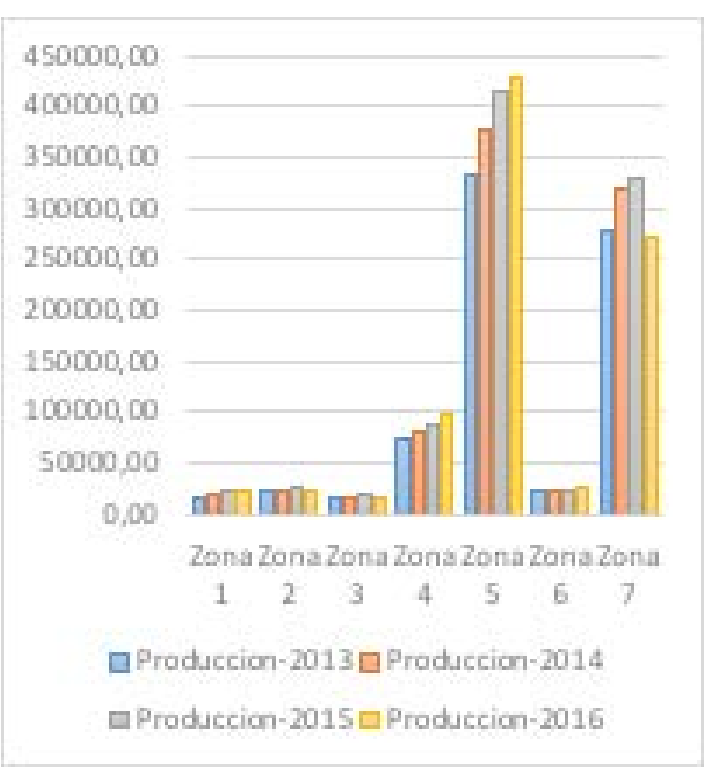

Figura 7. Producción total por Zona de Banano, Café y Cacao, 2013-2016

Fuente: Banco Central del Ecuador (2017)

En la Figura 8 la comparación corresponde a las zonas destacadas en volumen de producción como la 4, 5 y 7 integradas por Santa Elena, Guayas, Bolívar, Los Ríos y Galápagos (Zona 5) comenzando un apogeo a partir del 2013 hasta el 2016, Guayas alcanza el 80\% del total de la zona 5, luego Los Ríos, 14\%; Santa Elena, 4\%; y Bolívar, 2\%, los factores que provocaron la cúspide se resume en las campañas de apoyo a las actividades agrícolas con la apertura de nuevas líneas de crédito y flexibilidades del sector externo que fomentaron la reactivación económica interna.

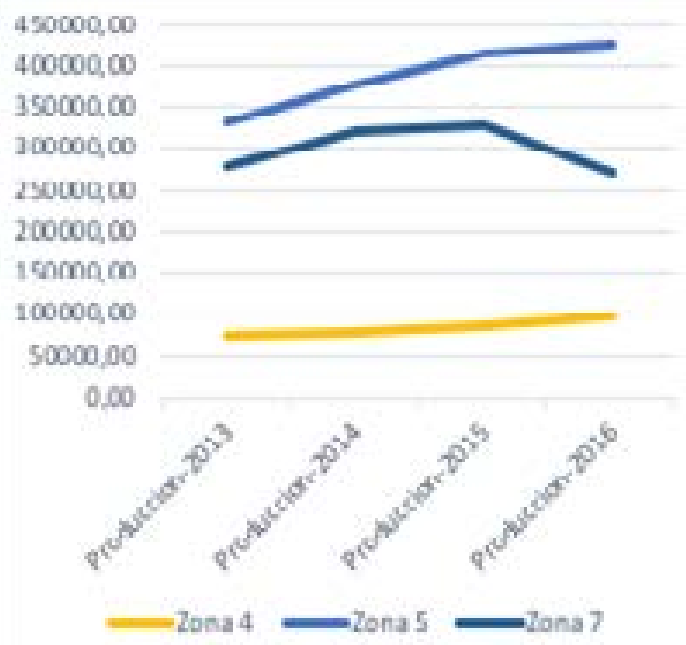

Figura 8. Producción de Banano, Café y Cacao: Zonas 4-5-7, 2013-2016

Fuente: Banco Central del Ecuador (2017) 
En la zona 7 conformada por las Provincias El Oro, Loja y Zamora Chinchipe su crecimiento fue desde el 2013 hasta el 2015, luego presentó una caída leve de la producción en el 2016, el desarrollo y la competitividad de la fruta de banano ha sido destacable en destinos europeos, pero la extensión territorial ha permitido que la producción sea fortalecida con la inversión de diferentes sectores, el café y el cacao son frutas de consumo nacional y de consumo externo, a pesar de la competitividad de otros países se destaca por las especificaciones de calidad; sin embargo, los niveles de producción decayeron en el último año de estudio por los instrumentos comerciales impuestos por otros países.

Y la zona 4, estructurada por Manabí y Santo Domingo, es la tercera que abarca mejor producción del 2013 al 2016, a pesar de su lento crecimiento es considerada zona especial de desarrollo económico, las oportunidades han destacado en proyectos de escala especialmente en el sector agrícola como el banano, café y cacao, además de políticas económicas focalizadas en ayudas crediticias para agricultores y exportadores.

El impacto económico de las zonas es destacable por la recaudación de ingresos y el aporte al Producto Interno Bruto nacional, a pesar de ser frutas de producción mundial, las de origen ecuatoriano son de mayor compra en los mercados externos.

Tabla 7.

Exportaciones de café, cacao y banano-Participación en el PIB

\begin{tabular}{lll}
\hline Año & $\begin{array}{l}\text { Exportaciones de Banano, Café y Cacao } \\
\text { (Miles de Millones USD) }\end{array}$ & PIB Total (Miles de Millones USD) \\
\hline 2013 & $1,095,461$ & $67,546,128$ \\
\hline 2014 & $1,188,275$ & $70,243,048$ \\
\hline 2015 & $1,301,110$ & $70,353,852$ \\
\hline 2016 & $1,292,189$ & $69,321,410$ \\
\hline
\end{tabular}

Fuente: Banco Central del Ecuador (2017)

En esta última Tabla 7 se observa que, en el 2013, las exportaciones alcanzaron un aporte al PIB de más de 67 mil millones de dólares, fortaleciéndose aún más en el 2015 con 70 mil millones de dólares, pese a una leve caída registrada en el 2016 por la disminución de los precios internacionales acontecida por una disminución de la demanda, pero no significó afectación en las exportaciones y ventas nacionales, es decir, el impacto económico ha sido positivo para la economía a través de este sector agrícola por el nivel de ingresos registrados.

\section{Conclusiones}

Los resultados analizados evidencian las necesidades de seguir en la especialización del sector agrícola, siendo Ecuador una economía pequeña con clima y tierras favorecedoras para la producción de materia prima necesaria para el consumo nacional y externo, mediante esta iniciativa se cumplió el objetivo de identificar la zonificación de mayor productividad referente al banano, café y cacao, así como la incidencia en la economía, los valores demostraron que la zona 5, 7 y 4 son las que mayor aportan en la renta nacional por los niveles de exportación, en 
el contexto histórico delimita a un país agroexportador con oferta de productos e importantes estándares de calidad.

Se evidencia, a su vez, que las decisiones de políticas económicas han sido encaminadas para generar progreso económico durante el tiempo de estudio, a pesar de haber pasado por caídas de precio del petróleo durante el 2015-2016. Se presentó una leve recesión económica que no afectó mayormente al sector del estudio desde la perspectiva de comercio exterior; sin embargo, presentó una caída del PIB en el 2013 y luego en el 2016.

Ante estos factores, la división zonal ha tomado un papel de iniciativa en articular y fomentar políticas públicas de inversión en sectores estratégicos para la economía ecuatoriana como es el sector agro, los porcentajes de crecimiento del PIB agropecuario se han mantenido en aumento durante el periodo planeado en la investigación, garantizando la sostenibilidad que brindan los bienes primarios por los programas de desarrollo del Estado.

Se concluye así que el Ecuador es un país proveedor de materia prima con potencial agrícola, posicionándose como uno de los principales socios comerciales latinoamericanos de mayor oferta exportable en países europeos, el período de estudio demostró el impacto económico de dichos productos en la economía nacional mediante el aporte del nivel de producción por zonas y las exportaciones en el Producto Interno Bruto. 


\section{Referencias}

Andrade, D., y Andrade, M. (2018). Análisis crítico sobre políticas públicas agropecuarias y el impacto económico en el Mercado de los úlitmos 10 años. Estudio de caso Chimborazo-Ecuador. Revista Observatorio Economía Latinoamericana. http://www.eumed.net/2/rev/oel/2018/02/politicas-publicas-agropecuarias. $\underline{\mathrm{html}}$

Banco Central del Ecuador (2017). Cuentas Nacionales Regionales. https://contenido.bce.fin.ec/documentos/ Estadisticas/SectorReal/CuentasProvinciales/Indice.html

Briones, C. (2018). Incidencia de factores determinantes en el sector agrícola en Ecuador: Banano, Cacao, Café y Palma africana. Período de estudio 2000-2017. Trabajo de Titulación previo a la obtención del título de Economista. Universidad Católica de Guayaquil, 119. http://repositorio.ucsg.edu.ec/bitstream/3317/11674/1/TUCSG-PRE-ECO-CECO-252.pdf

Cadena, P., Rendón, R., Aguilar, J., Salinas, E., De la Cruz, F., y Sangerman, D. (2017). Métodos cuantitativos, métodos cualitativos o su combinación en la investigación: un acercamiento en las ciencias sociales. Revista Mexicana de Ciencias Agrícolas, 8(7), 1603-1617.

Cazu, P. (2004). Categorización y Operacionalización. Obtenido de Guia Metodológica de la Investigación.

Coria P, Torres H, Z., y Roman, I. (2014). Propuesta de metodología para elaborar una investigación científica en el área de Administración de Negocios.

El Productor (2017). Producción nacional de banano. https://elproductor.com/estadisticas-agropecuarias/produccion-nacional-de-banano/

Food and Agriculture Organization of the United Nations (FAO). (2017). El sector Agrícola y el crecimiento económico. http://www.fao.org/3/y5673s/y5673s05.html

Gonzabay, R. (2013). Cultivo del banano en el Ecuador. afese, 1-30. Recuperado de: http://www.revistaafese. org/ojsAfese/index.php/afese/article/view/317

Instituto Nacional de Estadísticas y Censos (2016). Encuesta Superficie y Producción Agropecuaria Continua ESPAC 2016. https://www.ecuadorencifras.gob.ec/documentos/web-inec/Estadisticas agropecuarias/espac/espac-2016/Informe\%20ejecutivo\%20ESPAC 2016.pdf

Loayza, F. (2018). Análisis de la cadena productiva del cacao ecuatoriano para el diseño de una política pública que fomente la productividad y la eficiencia de la producción cacaotera período 2007-2016. Disertación de grado previo a la obtención del título de Economista. Pontificia Universidad Católica del Ecuador.

Ministerio de Agricultura, Ganadería, Acuacultura y Pesca (MAGAP). (2017). La Política Agropecuaria Ecua- 
toriana. Hacia el desarrollo territorial rrural sostenible 2015-2025: El sector agropecuario ecuatorianao, análisis histórico y prospectiva a 2025. http://www.competencias.gob.ec/wp-content/uploads/2017/05/ 01PPP2016-POLITICA01.pdf

Pino, S., Aguilar, H., Apolo, A., y Sisalema, L. (2018). Aporte del sector agropecuario a la economía del Ecuador. Análisis crítico de su evolución en el período de dolarización. Años 2000 - 2016. Revista Espacios, 1-11.

Plata, D. J. (2019). Cómo Hacer Un Perfil Proyecto De Investigación Científica.

Secretaría Nacional de Planificación y Desarrollo del Ecuador (SENPLADES). (2012). Senplades. La planificación: un instrumento para consolidar la regionalización. https://www.planificacion.gob.ec/wp-content/ uploads/downloads/2012/08/La-Planificaci\%c3\%b3n.pdf

Secretaria Nacional de Planificación y Desarrollo del Ecuador (SENPLADES). (2015). Senplades. Agenda Zonal Zona 1- Norte Provincias de Esmeraldas, Imbabura, Carchi, Sucumbíos 2013-2017. https://www.planificacion.gob.ec/wp-content/uploads/downloads/2015/11/Agenda-zona-1.pdf

Secretaria Nacional de Planificación y Desarrollo del Ecuador (SENPLADES). (2018) Senplades. Plan Estratégico Senplades 2014-2017. https://www.planificacion.gob.ec/wp-content/uploads/downloads/2018/10/ Plan-Estrategico-Senplades-2014-2017.pdf

Trívez Bielsa, F. J. (2015). Introduccion a la econometria. Santiago, Chile. https://www.marcialpons.es/libros/ introduccion-a-la-economia/9788436838077/

Villavicencio, J. (2013). Introducción a Series de Tiempo. Costa Rica. 
Copyright (c) 2020 Lady Andrea León-Serrano, Amparo Marisol Matailo-Pinta, Aida Andreina Romero-Ramón y Cecilia Alexandra Portalanza-Chavarría

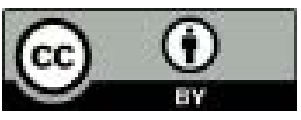

Este texto está protegido bajo una licencia internacional Creative Commons 4.0.

Usted es libre para Compartir-copiar y redistribuir el material en cualquier medio o formato

- y Adaptar el documento - remezclar, transformar y crear a partir del material-para cualquier propósito, incluso para fines comerciales, siempre que cumpla las condiciones de Atribución. Usted debe dar crédito a la obra original de manera adecuada, proporcionar un enlace a la licencia, e indicar si se han realizado cambios. Puede hacerlo en cualquier forma razonable, pero no de forma tal que sugiera que tiene el apoyo del licenciante o lo recibe por el uso que hace de la obra.

Resumen de licencia - Texto completo de la licencia 